\title{
Keramik- und CAD / CAM-Protagonist erhielt Professur
}

Seine zahllosen Poster, Publikationen, Reviews und Referate zur vollkeramischen Restauration sowie zur CAD/ CAM-Technik in der zahnärztlichen Prothetik reihen sich zu einem „Vademecum der Keramik“ und findet in $\mathrm{zu}^{-}$ nehmendem Maße Beachtung in der internationalen Zahnärzteschaft.

Begonnen an der Poliklinik für Zahnärztliche Prothetik der Ludwig-MaximiliansUniversität in München unter der Leitung von Prof. Dr. Wolfgang Gernet und Promotion bei Prof. Dr. Peter Pospiech, entwickelte der bisherige Privat-Dozent Dr. Florian Beuer in den Jahren von 2002 bis heute ganz wesentliche Forschungsinitiativen mit dem Ergebnis, dass für die Vollkeramik neue Indikationen in der Prothetik und in der Implantologie erschlossen wurden. So entstanden unter seiner Teamleitung konkrete, praxisgerechte Lösungen für Kronen und Brücken aus Zirkoniumdioxidkeramik, die computergestützte Fertigung von Verblendschalen für $\mathrm{ZrO}_{2}$ Gerüste, die Nutzung von Titan-stabilisierten ImplantatAbutments aus $\mathrm{ZrO}_{2}$ und viele Studienergebnisse zur klinischen Langzeitbewährung von vollkeramischen Rekonstruktionen. Federführend am „Münchener Implantatkonzept" hat Beuer ganz wesentlichen Anteil an diesem praxistauglichen Therapieverfahren.

Internationale Erfahrungen sammelte Beuer als Visiting Professor am Pacific Dental Institute in Portland/USA bei John Sorensen DMD, PhD. Durch seine Fähigkeit zur Integration wurde er 2009 zum Vizepräsident der Deutschen Gesellschaft für Ästhetische Zahnheilkunde (DGÄZ) gewählt und wurde 2010 zum zertifizierten Spezialisten der Deutschen Gesellschaft für Prothetische Zahnmedizin und Biomaterialien (DGPro) für zahnärztliche Prothetik ernannt.

In der Habilitationsschrift, verfasst im Jahr 2008, thematisierte Beuer seine Erfahrungen unter dem Titel „Untersuchungen zur Verbesserung der klinischen Langzeitprognose von Restaurationen aus Zirkoniumdioxid“. Diese richtungsweisende Arbeit und die Anerkennung der zahlreichen Initiativen und Projekte wurden nach 4 anstelle der üblichen 6 Jahren Karenzzeit (nach dem bayerischen Hochschulpersonalgesetz) belohnt: Am 27. Februar 2014 wurde PD Dr. Florian Beuer, knapp 40-jährig, zum apl-Professor ernannt. Wir gratulieren herzlich.

Nach einer Pressemitteilung der Arbeitsgemeinschaft für Keramik in der Zahnheilkunde e.v.

\section{Design-Preise für zahnmedizinische Technik}

Die Dürr Dental AG wurde jüngst für ihr technisches, optisches und gestaltendes Design innovativer medizinischer Produkte mit dem Good Design Award durch das „Chicago Athenaeum Museum of Architecture and Design“ prämiert. Der Good Design Preis 2013 ging an das Sterilisationsverpackungsgerät Hygopac Plus. Bei den ebenfalls ausgezeichneten Speicherfolienscannern VistaScan Mini View und VistaScan Combi

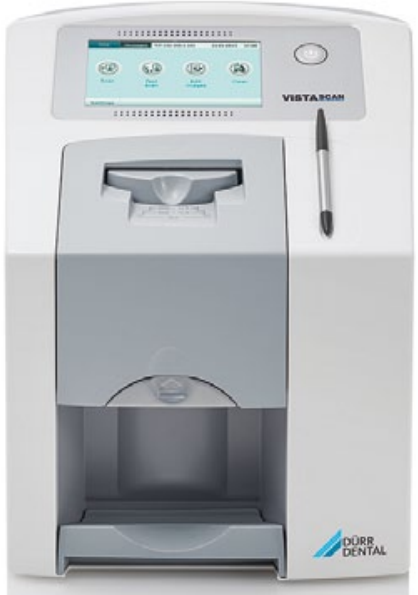

View handelt es sich um die neueste digitale Technologie für das Röntgen im Mundraum. Ihre bedienungsfreundliche Handhabung und formschöne Gestaltung wurde für den Good Design Award

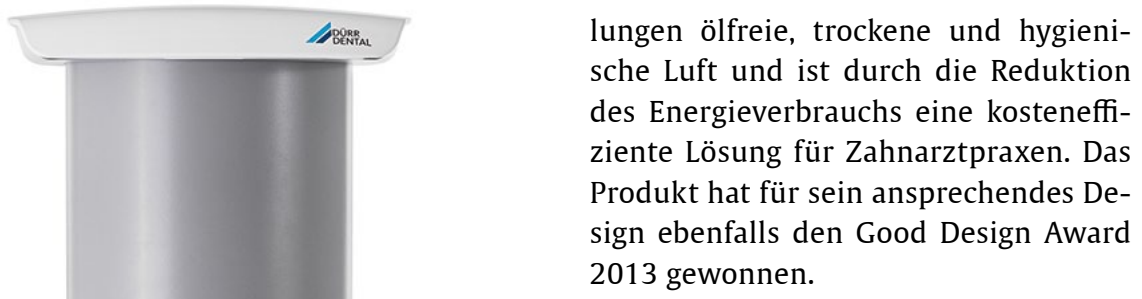

Der Good Design Award wurde 1950 in der amerikanischen Metropole Chicago gegründet und ist weltweit die älteste und meist beachtete Auszeichnung für exzellentes Design.

Nach einer Pressemitteilung der Dürr Dental AG, Bietigheim-Bissingen Internet: www.duerrdental.com 


\section{von Praxen und Laboren}

Die Erfolgsgeschichte der VITA Zahnfabrik begann vor 90 Jahren. Am 17. Juli 1924 in Essen gegründet und seit 1943 in Bad Säckingen beheimatet, wird das Familienunternehmen heute in 3. Generation geführt. Mit der Entwicklung des Helios Zahns in den 1920er Jahren wurde der Grundstein gelegt für die VITA Kompetenzfelder Künstliche Zähne, Farbe und Keramik. Gleichzeitig resultierte die Weiterentwicklung der ersten Farbringe aus der Gründungszeit 1955 in der Lumin-VACUUM Farbskala, die Basis für das 1983 eingeführte Farbsystem VITAPAN classical A1-D4. Seit 1998 steht zusätzlich das VITA SYSTEM 3DMASTER zur Verfügung. Beide Systeme haben sich als internationaler Standard etabliert: 4 von 5 Farbwerten weltweit werden mit ihnen bestimmt. Wie ton- angebend VITA auch im Bereich Keramik ist, wird u.a. durch die Übernahme des Produktnamens VMK (VITA Metallkeramik) von 1962 als allgemeine Bezeichnung in den Sprachgebrauch belegt. Die Entwicklung der 1. VITA-Dentalkeramik zur Herstellung von individuellem Zahnersatz geht auf das Jahr 1930 zurück. Darüber hinaus ist VITA Pionier auf dem Sektor der dentalen CAD / CAM-Materialien. Bereits 1985 wurde erstmals ein Patient mit einem Inlay versorgt, das aus einer Keramik bestand, die der der späteren VITABLOCS sehr ähnlich war. Dank diverser Systempartner sind VITA-Materialien heute mit vielen dentalen CAD/CAMSystemen bearbeitbar.

Nach einer Pressemitteilung der VITA Zahnfabrik H. Rauter GmbH \& Co. KG, Bad Säckingen

\section{Neue Partnerschaft}

\section{Globales Portfolio gestärkt}

GC stärkt sein Angebot in der globalen CAD/CAM-Sparte und schließt eine zukunftsträchtige strategische Partnerschaft mit dem DentaltechnologieUnternehmen Sirona. „Wir freuen uns, bei der Herstellung von CAD/CAM-Blöcken für CEREC und inLab, etwa aus Kompositen oder anderen Restaurationsmaterialien, ab sofort mit Sirona zusammenzuarbeiten“, so der Executive Vice President Henri Lenn. Sirona entwickelt, produziert und vermarktet ein vollständiges Programm an Dentalprodukten, wie z.B. CAD/CAM-Restaurationssysteme, digitale, intraorale Panorama- und 3DAbbildungssysteme, Zahnbehandlungszentren und Handstücke. GC ist Marktführer bei den Glasionomermaterialien und darüber hinaus in den Produktbereichen Komposite, Verblendkeramiken und Ad-

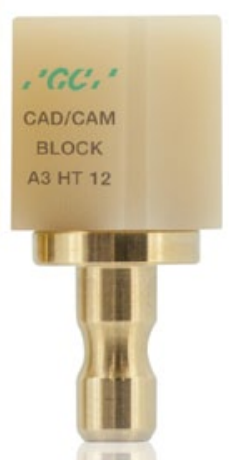

häsivsystemen. Um den wachsenden Bedarf der Zahnärzte an Dentaltechnologie in Verbindung mit qualitativ hochwertigen Materialien zu decken, haben sich die beiden genannten Dentalunternehmen zu einer Partnerschaft entschlossen. Sirona profitiert dabei von der Marketing-Unterstützung im schnell wachsenden asiatischen Markt, während GC den weltweiten $\mathrm{Zu}$ gang zu Sironas bestehenden CAD/CAMAnwendern erhält. „Dank unserer gemeinsamen Vision und der übereinstimmenden Grundwerte begrüßen wir die Zusammenarbeit und freuen uns darauf, die Standards in der Zahnmedizin weiter zu erhöhen“, fasst Henri Lenn zusammen.

Nach einer Pressemitteilung der GC EUROPE N.V., B-Leuven

Internet: www.gceurope.com

\section{Jubiläum}

145 Jahre VDW

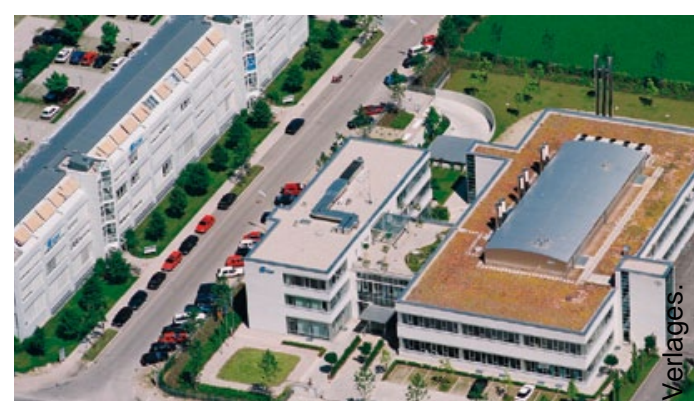

Die 1869 in München gegründete Firma C. W. Zipperer begann mit der Herstellung feinmechanischer Teile für Uhrmacher und wurde bald darauf der 1. europäische Hersteller von Instrumenten für die Wurzelkanalbehandlung. Zipperer ist der älteste Teil des heutigen Unternehmens VDW GmbH. Der Zahnarzt J. Beutelrock, Sohn eines Uhrmachers, gründete 1885 seine „Spezialfabrik eigener Erfindungen“, hauptsächlich für Instrumente zur „Ausschachtung des Wurzelkanals“. Beide Unternehmen haben ihre Produkte schon zur Jahrhundertwende auf den Weltmärkten vertrieben. 1919 schließlich starteten die ANTÆOS-Werke. Diese 3 Münchner Unternehmen waren lange Konkurrenten. Sie haben ihr Instrumentarium stetig erweitert und verbessert. ANTÆOS hat später die Konkurrenten ZIPPERER und BEUTELROCK übernommen und 1972 zu „Vereinigte Dentalwerke“ fusioniert, jetzt kurz VDW GmbH. Die 3 Marken werden bis heute zur Fortführung jahrzehntealter Vertriebspartnerschaften beibehalten. An der rasanten Entwicklung der Endodontie in den letzten 15 Jahren ist VDW maßgeblich beteiligt. Investitionen in Forschung und Entwicklung sowie enge Zusammenarbeit mit Wissenschaft und Praxis wurden in neue Konzepte umgesetzt, die die Arbeit des Zahnarztes vereinfachen, verbessern und angenehmer gestalten.

Endo in der DNA: 145 Jahre Erfahrung und konsequente Spezialisierung auf Endodontie haben dem Unternehmen ein starkes Wachstum ermöglicht. Diese Stärke wird konsequent zur Weiterentwicklung endodontischer Konzepte nach dem Motto „Endo Einfach Erfolgreich“ genutzt.

Nach einer Pressemitteilung der VDW GmbH, München

Internet: www.vdw-dental.com 